\title{
Novel diamond X-ray crystal optics for synchrotrons and X-ray free-electron lasers
}

\author{
Stanislav Stoupin*
}

\begin{abstract}
The most common applications of diamond crystals in X-ray optics are high-heat-load monochromators for synchrotron beamlines and phase retarders for polarization control. Here, less common applications of diamond at the frontier of X-ray crystal optics are reviewed and summarized. These include a sub-meV-bandwidth X-ray monochromator with high spectral efficiency [1] and all-diamond optical assemblies for beam-multiplexing doublecrystal monochromator at the Linac Coherent Light Source [2]. Also, novel applications for the realization of fully coherent hard X-ray sources are discussed, such as, diamond crystal optics for self-seeding of hard X-rays in the Linac Coherent Light Source [3,4] and Bragg mirrors for the highly anticipated X-ray free-electron laser oscillator $[5,6]$. These examples present diamond as a material for the next generation of X-ray optics, optics which can provide unique characteristics and capabilities to modern X-ray sources. In addition, details of practical importance on fabrication and characterization methods of diamond crystals with the suitable quality are presented.
\end{abstract}

Keywords: HPHT, diamond crystal, defects, X-ray optics, XFEL

\section{Introduction}

The main tasks of X-ray optics are tailoring photon flux, direction of propagation, angular spread, polarization and energy bandwidth of radiation generated by an X-ray source to desired specifications, which enables

${ }^{*}$ Corresponding author at: 9700 S. Cass Ave, Argonne, IL 60527, USA. Tel: +1-630$252-8548$

Email address: sstoupin@aps.anl.gov (Stanislav Stoupin ) 
measurements of particular effects and/or properties of matter. As radiation wavelengths approach the characteristic size of an atom (Angstrom) measurement techniques enter the domain of hard X-rays. In this regime, diffraction of X-rays on a crystal lattice is commonly utilized for tailoring of the radiation properties. A variety of single crystals with nearly perfect lattice are employed for this purpose. Examples of such crystals (X-ray optical crystals) are $\mathrm{Si}, \mathrm{Ge}, \mathrm{C}$ (diamond), $\mathrm{SiO}_{2}$ (quartz), $\mathrm{Al}_{2} \mathrm{O}_{3}$ (sapphire), $\mathrm{LiF}$ and others. Among them, diamond plays a special place role in manipulation of $\mathrm{X}$-rays generated by high-brilliance sources, modern synchrotrons and X-ray free-electron lasers (XFELs). Diamond has the highest thermal conductivity of any known material and a record high reflectivity in Bragg diffraction, the highest radiation hardness and elastic modulus among the X-ray optical crystals, a practical, low X-ray absorption and thermal expansion coefficients.

Diamond as a Bragg crystal is indispensable in the following applications: beam-multiplexing monochromators for X-ray freeelectron lasers (XFEL) $[2,7,8]$, Bragg mirrors for X-ray freeelectron laser oscillators (XFELO) $[5,6,9,10]$, and monochromators for self-seeding of XFELs $[3,4,11]$. The use of diamond as a diffracting crystal in high-heat-load monochromators (e.g., $[12,13$, 14]) has the advantage of reduction in operating costs (water cooling vs. cryogenic cooling of $\mathrm{Si} / \mathrm{Ge}$ crystals). In high-resolution Xray optics diamond can yield substantial improvement in the spectral efficiency $[1,15,16]$. Also, single crystal diamond is among the best candidate materials for front-end wavefront-preserving vacuum windows [17] and phase retarders $[18,19,20,21]$.

In this work, novel applications of diamond in hard X-ray optics are reviewed in conjunction with state-of-the-art crystal fabrication and processing methods, and physical properties of this remarkable material. The paper is organized as follows. First, state-of-the-art quality of single crystal diamond is discussed attained using high-temperature high-pressure (HPHT) synthesis methods. Second, practical methods of X-ray diffraction characterization are presented. Finally, the novel applications of diamond in X-ray crystal optics are discussed. Additional insights to these applications and perspectives on further development of diamond X-ray crystal optics are offered. 


\section{Fabrication of diamond X-ray crystal optics}

High crystalline quality of diamond is attained under equilibrium crystal growth conditions which require high pressures $(P \gtrsim 5 \mathrm{GPa})$ and high temperatures $(T \gtrsim 1500 \mathrm{~K})$ (e.g., [22]). The largest size of the as grown crystals can be up to $12 \mathrm{~mm}$ in diameter [23]. The lowest concentration of crystal lattice defects is attained in the (001) growth sector in the upper part of the crystals grown in HPHT apparatus from graphite using seeds with high crystalline quality. Boron and nitrogen impurity concentrations less than 0.1 ppm are readily demonstrated for this growth sector. Under the conventional classification of diamond based on optical absorption properties such crystals fall into the type IIa or type IIb categories [24] (depending on the prevalence of nitrogen or boron as the main impurity).

Figure 1 summarizes production of high-quality crystal plates from as grown HPHT diamond crystals. Several as grown crystals are shown in Fig. 1(a). A topograph (X-ray diffraction image) of a cross-section of one of these crystals is shown Fig. 1(b) revealing a particular distribution of crystal defects. Growth sectors are identified as crystal regions separated by dark stripes (stacking faults). The largest (light-colored) central portion of the crystal is the (001) growth sector. Crystal plates of (001) orientation are obtained by slicing the crystal along the horizontal lines. Topographs of the resulting plates reveal distribution of crystal defects that reflects the structure of the corresponding cross section of the as grown crystal. The plate with the largest region of high-crystal quality is obtained from the topmost portion of the crystal. A photograph of one of such plates is shown in Fig. 1(c). The visual appearance of a high-quality synthetic diamond crystal is similar to that of clear glass or plastic (crystal defects are not visualized). Fig. 1(d) shows an X-ray topographs of the same plate where crystal defects are clearly seen on the edges. The central part remains nearly defect-free.

Defects in the crystal lattice dramatically affect Bragg reflectivity due to presence of the local strain fields. These tend to destroy the coherent superposition of the waves in Bragg diffraction. A substantial reduction in the reflectivity and a broadening of the reflectivity curves is the common outcome.

Chemical Vapor Deposition (CVD) method is now commonly used to synthesize single crystals of diamond using polished high-quality HPHT crystals as a substrate. Most single crystals produced by the CVD method suffer from high dislocation density resulting from the surface defects in the sub- 

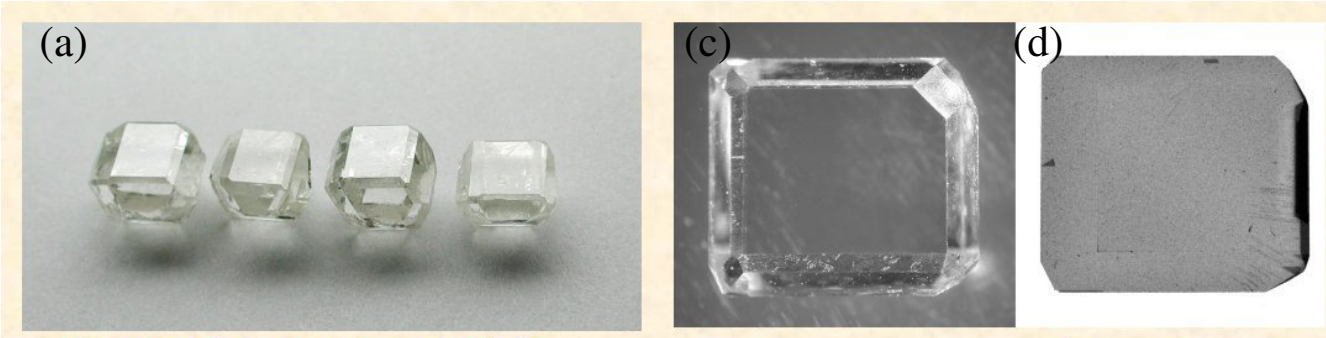

\section{(b)}

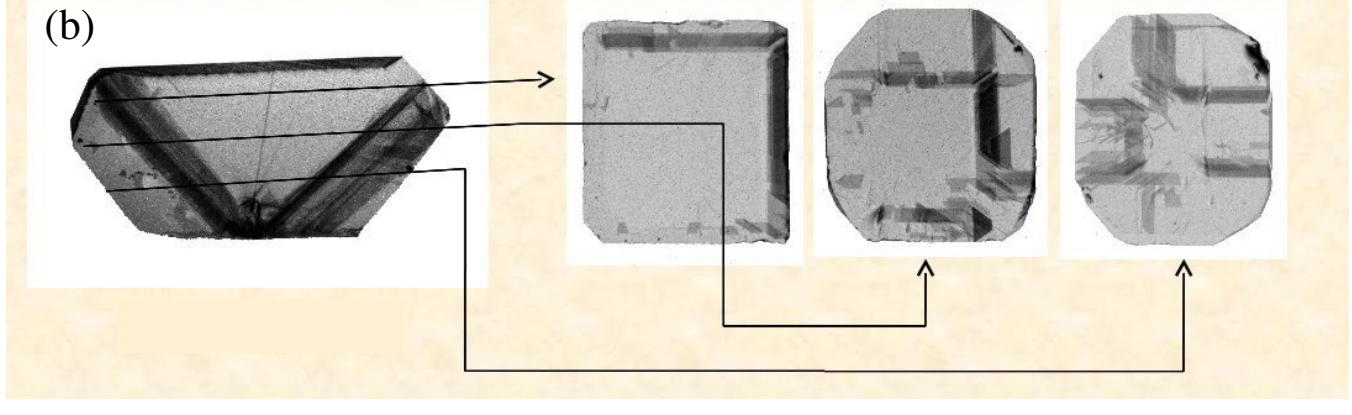

Figure 1: Production of high-quality diamond crystal plates (a) as grown HPHT crystals; (b) a topograph of a cross section of one of the as grown crystals and topographs of (001) plates originating from different sections as shown by the arrows; (c) a photograph of high-quality crystal plate obtained from the topmost portion of the as grown crystal, and (d) X-ray topograph of the same crystal plate. The images shown are courtesy of S.A. Terentyev (Technological Institute of Superhard and Novel Carbon Materials, Russia). 
strate that propagate in the volume of the CVD crystal during the deposition process. These general considerations, however, should not be considered as a verdict that prevents applications of CVD diamond in X-ray crystal optics. The progress on the production of high-quality CVD single crystals is ongoing (e.g., [25]) and might eventually yield crystals of acceptable quality.

\section{X-ray diffraction characterization}

Various techniques for imaging of diamond using X-rays have been recently reviewed [26]. A crystal for applications in X-ray crystal optics is assumed to be of single domain (absence of mosaicity), having well-defined working surfaces (i.e., polished or cleaved) and containing a region with low density of crystal defects of a suitable area size $\left(\simeq 1 \times 1 \mathrm{~mm}^{2}\right.$ or greater).

A very robust method for selection of high-quality crystals is X-ray whitebeam topography using a polychromatic (white) collimated X-ray beam with a large cross section and high brilliance available at a bending magnet beamline of a synchrotron source. The convenience of this method is due to availability of several reflections in one image conveniently obtained by a short exposure of the entire crystal (or its large portion) to the white beam. The most common experimental arrangement is the transmission geometry (owing to low X-ray absorption of diamond). The reflected monochromatic beams (Laue reflections) are projected onto an X-ray film placed behind the crystal. Each projection representing a diffraction image of the crystal is referred to as white-beam X-ray topograph of a corresponding $h k l$ reflection. The spatial resolution of the method is limited by the emulsion grain size of X-ray films, which can be as small as $\approx 1 \mu \mathrm{m}$.

Defects in crystals of dimensionality 1 (dislocations), 2 (stacking faults) and 3 (inclusions) are readily observed as well as surface deformations (e.g., due to imperfect crystal polishing). For a detailed discussion of various mechanisms of image contrast in white-beam X-ray topography of diamond the reader is referred to [27].

Figure 2 shows white-beam X-ray topographs of selected (001) type IIa synthetic diamond plates from different HPHT diamond producers: a 0.4mm-thick plate by Element 6 (a), a 0.4-mm-thick plate by Sumitomo Electric (b) and a 1.0-mm-thick plate by Technological Institute of Superhard and Novel Carbon Materials (TISNCM) (c). The topographs were obtained in the Laue (transmission) geometry from the 131 reflections. 

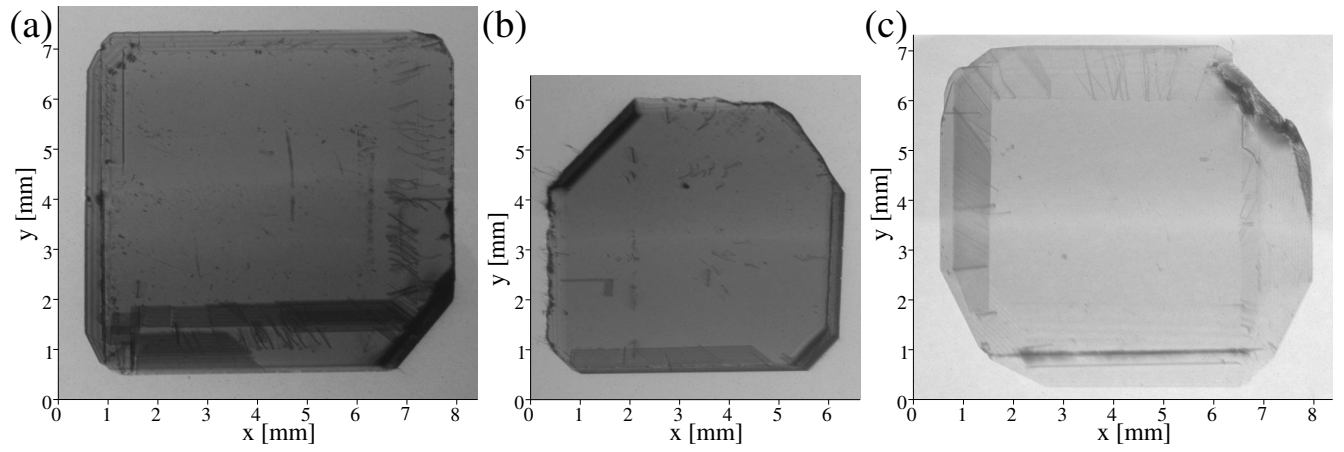

Figure 2: White-beam X-ray topographs of selected (001) type IIa synthetic diamond plates from different HPHT diamond producers: a 0.4-mm-thick plate by Element 6 (a), a 0.4-mm-thick plate by Sumitomo Electric (b) and 1.0-mm-thick plate by TISNCM (c). The topographs were obtained in the Laue (transmission) geometry from the 131 reflections.

The topographs are characterized by low dislocation density, only a few micro-inclusions and absence of stacking faults in the central part of the plates (at least $4 \times 4 \mathrm{~mm}^{2}$ in size). This observation suggests that the plates are obtained from the topmost part of the (001) growth sector. The crystal manufactured by TISNCM is remarkable due to the fact that the nearly defect-free region described above extends to the depth of $\simeq 1 \mathrm{~mm}$, which can yield close-to $100 \%$ reflectivity in backscattering Bragg diffraction even for very hard X-rays with photon energies above $20 \mathrm{keV} \mathrm{[6].}$

Burns et al. [17] present an excellent overview and a comprehensive classification of diamond crystal quality for applications in X-ray optics. Under this classification the novel applications of diamond summarized in this work rely on crystals that fall into class 3. This class is characterized by the working crystal belonging to a single dislocation-free growth sector and absence of growth striations (microinhomogeneities).

X-ray topography studies of state-of-the-art HPHT diamond crystals combined with high-resolution X-ray diffraction measurements $[2,4,5,6,17,28,29,23]$ suggest that the portion of a diamond crystal of various orientation originating from the (001) growth sector represents the optimal working region for diamond X-ray optics. Thus, crystals with surface orientation close to (001) have the largest defect-free working regions. For other orientations (e.g., (111)) the size of the available defect-free region is somewhat 
reduced as will be shown in one of the examples below.

\section{Applications of single crystal diamond in hard X-ray optics}

\subsection{General remarks}

Traditional applications of single crystal diamond in hard X-ray optics include high-heat-load monochromators (e.g., $[12,13,14]$ ) and phase retarders for polarization control/analysis (e.g., [18, 19, $20,21]$ ). In addition, CVD diamond (primarily polycrystalline) is explored by the synchrotron/XFEL community and used for fabrication of X-ray refracting lenses $[30,31,32]$, zone plates [33, 34], and implementation of vacuum windows (e.g., [35]).

One of the main advantages of using diamond crystals for high-heat-load monochromators is the applicability of water cooling for thermal stabilization of the diffracting crystal at undulator beamlines of third-generation synchrotron sources. This yields a reduction in operating cost compared to a more traditional solution of using liquid-nitrogen-cooled Si crystals. However, there are disadvantages such as the small size of high-quality diamond crystals and smaller energy bandwidths of low-order diamond $h k l$ reflections compared to the bandwidths of the corresponding reflection in Si. For example, the relative intrinsic energy width of the 111 reflection in diamond is $\varepsilon_{\mathrm{C}} \simeq 5.7 \times 10^{-5}$ (for a monochromatic wave with $\sigma$-polarization). For the Si 111 reflection this value is $\varepsilon_{\mathrm{Si}} \simeq 1.3 \times 10^{-4}$, which results in a higher photon flux delivered by the monochromator. To a good approximation the relative intrinsic energy bandwidth is independent on the photon energy for an incident wave with $\sigma$-polarization (typical Bragg diffraction geometry at beamlines of third-generation synchrotron sources). Therefore, the application of diamond in high-heat-load monochromators is justified for beamlines specializing in high-energy-resolution X-ray spectroscopies.

Phase retarders are used for production of circularly polarized X-rays which are a powerful probe of angular momenta in magnetic materials. Transmission X-ray phase retarders which utilize Bragg or Laue forward-diffracted beams offer simplicity and high efficiency provided that a nearly perfect crystal with low photoabsorption is utilized [19]. Diamond is one of the most widely used options for phase retarders.

The following discussion will be focused on other, less common applications of single crystal diamond at the frontier of X-ray optics. In these 
applications, diamond is either indispensable as a Bragg crystal or yields unsurpassed performance parameters due to its remarkable physical properties.

\subsection{Monochromators for self-seeding of hard X-rays in XFEL}

In this application a diamond crystal serves as a monochromator to select a narrow spectral portion of X-rays produced by a first series of XFEL undulators to seed a subsequent series of undulators [3]. The resulting radiation has a much greater temporal coherence compared to that available in the conventional XFEL operation mode known as self-amplified spontaneous emission (SASE). A 40-50 times reduction in the relative energy bandwidth was demonstrated in comparison with SASE at photon energies of 8-9 keV.

This demonstration of a fully coherent high-brilliance X-ray source was preceded by several technical developments. One of the major project milestones was the development of the self-seeding diamond optics. For this particular application a diamond with high crystal quality was indispensable. The use of diamond was dictated by its low photoabsorption (and thus greater seeding power in forward diffraction) as well as by high stability in the XFEL beam (due to high radiation hardness, high thermal conductivity and high elastic modulus). Special 100- $\mu$ m-thick (001) diamond plates with high crystal quality and trapezoidal shape were manufactured along with graphite holders for these plates to avoid radiation damage and to minimize mountinginduced strain in the crystals $[4,11]$. Defects in these diamond plates were characterized by white-beam synchrotron X-ray topography. Double-crystal

topography using a laboratory $\mathrm{Cu} K_{\alpha}$ source was utilized for diagnostics and minimization of strain in the mounted diamond plates.

\subsection{Beam-multiplexing monochromators}

Utilization of X-ray monochromators for beam multiplexing using low absorption crystals, such as diamond, was pioneered at the TROIKA beamline at the ESRF (European Synchrotron Radiation Facility, Grenoble, France) [36, 37, 12]. This beam multiplexing approach gains even more importance for hard X-ray free-electron lasers, which recently redefined the frontiers of X-ray sciences $[38,39,3]$. Using a single straight electron trajectory of an XFEL, multi-user operations cannot be achieved as is commonly accomplished at storage-ring-based synchrotron sources. However, multiplexing can be performed by means of X-ray optics, which enables simultaneous delivery of portions of the XFEL beam to several experiments. This yields an increase 


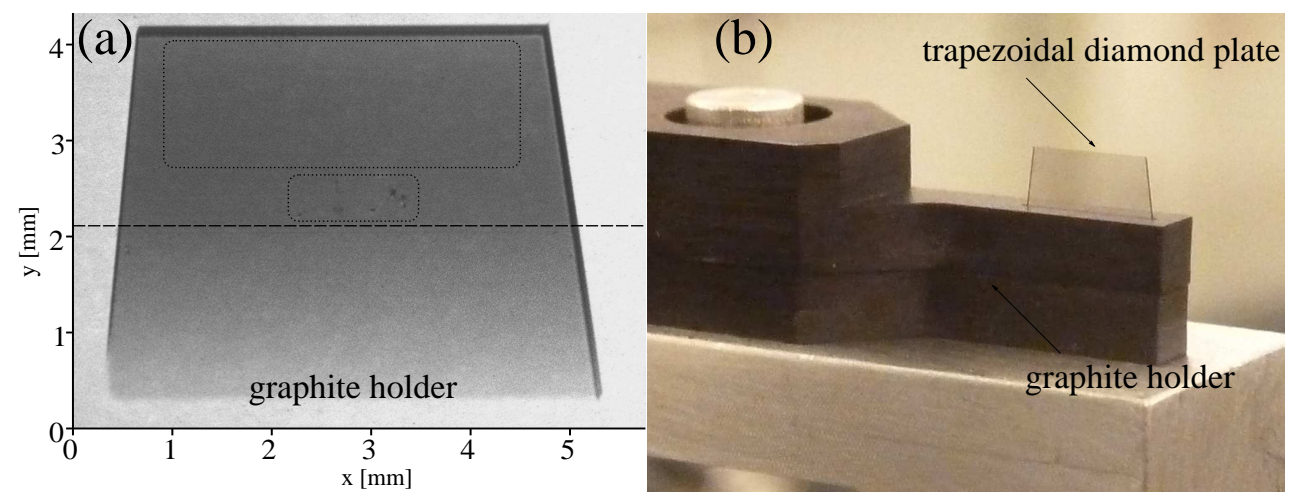

Figure 3: Diamond optics for self-seeding of hard X-rays in XFEL: (a) White-beam X-ray topograph of the 100- $\mu \mathrm{m}$-thick trapezoidal diamond plate with (001) surface orientation. The image obtained from 311 diamond reflection in Laue geometry. The large rectangular area is a defect-free working region. Defects can be noticed in the small rectangular central region. The plate was inserted into the graphite holder as shown in (b).

in the total number of performed experiments and thus reduces the high XFEL operating cost per experiment.

A scheme of a double-crystal beam-multiplexing monochromator in Bragg reflection geometry is shown in Fig. 4 . If the first crystal is made sufficiently thin, the beam transmitted through it can be used in a parallel experiment downstream. For beam multiplexing at an XFEL appropriate levels of Xray transmittance (at photon energies of $4-10 \mathrm{keV}$ ) can be achieved using crystal thicknesses of $\approx 5-10 \mu \mathrm{m}$ in the case of $\mathrm{Si}$ and $50-100 \mu \mathrm{m}$ in the case of diamond due the lower X-ray absorption of the latter material. The greater crystal thickness and Young's modulus for diamond ensure a substantially greater stiffness of the crystal. Diamond crystals with thicknesses of $\simeq 100 \mu \mathrm{m}$ as the first crystal in the beam-multiplexing double-crystal monochromator were found to be substantially more stable in the XFEL beam compared with the ultrathin Si crystals [7].

To maximize the flux in the monochromatic branch the lowest possible order of the Bragg reflection is preferred due to its greater intrinsic energy bandwidth (the 111 reflection in crystals with diamond structure). Because of the small divergence ( $\lesssim 1 \mu \mathrm{rad}$ rms) of the XFEL beam its wavefront is particularly sensitive to imperfections of X-ray optics. Disturbances of the wavefront introduced by the optics should be much less or at least comparable to the beam divergence, which is a challenging requirement, especially for diamond (111) crystals prone to crystal defects. 


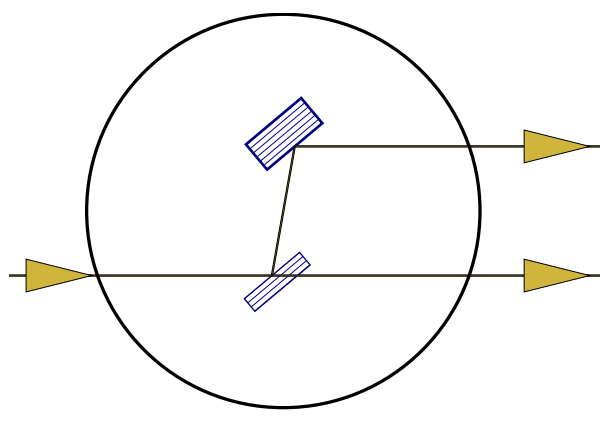

Figure 4: Scheme of a double-crystal beam-multiplexing monochromator in Bragg reflection geometry. The first crystal is sufficiently thin to avoid substantial losses in the transmitted branch due to photoabsorption.

Along with the presence of intrinsic defects, a mounting-induced crystal strain is another main factor that leads to deterioration of the crystal diffraction performance, which disturbs the radiation wavefront. The mounting solution developed for the diamond (001) self-seeding XFEL monochromator summarized in the previous subsection was found to show limitations in heat transfer between the crystal plate and the graphite holder where the plate was loosely mounted [7]. Thus, implementation of an XFEL beam-multiplexing diamond monochromator crystal fabrication and mounting required substantial revisions.

To address the new requirements 100- $\mu$ m-thick diamond (111) crystals plates with $5 \times 2 \mathrm{~mm}^{2}$ nearly defect-free regions were fabricated and alldiamond assemblies were developed where the crystal plates were mounted on polished CVD diamond substrates [2]. The mounting strain was optimized using developed thin CVD diamond springs with strain relief cuts made by laser. Distribution of strain in the crystal plates was characterized using rocking curve imaging in the double-crystal configuration.

Figure 5 summarizes the design, implementation and characterization of the all-diamond assembly for the 100- $\mu \mathrm{m}$-thick diamond plate. Main design features, a white-beam topograph showing distribution of defects in the diamond plate and microscope images of the all-diamond assembly are shown in Figures 5(a-e). 
(a)

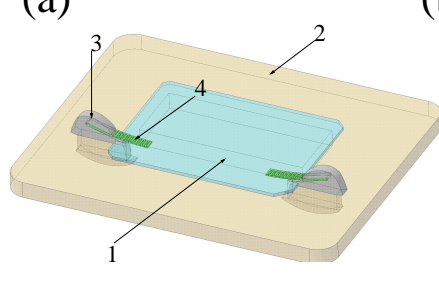

(b)

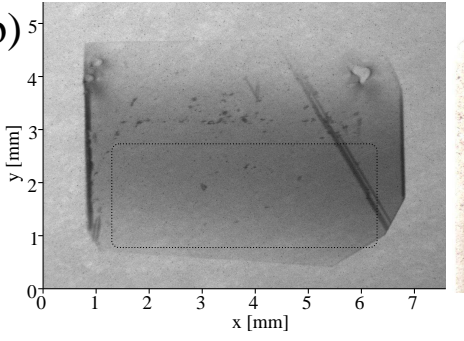

(c)

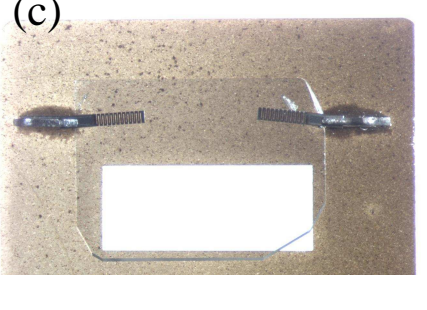

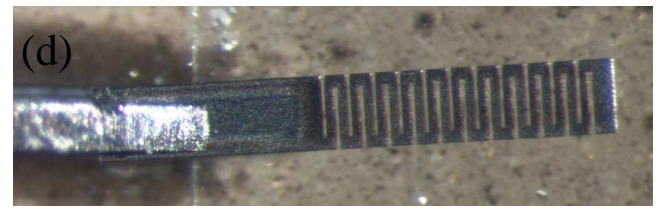

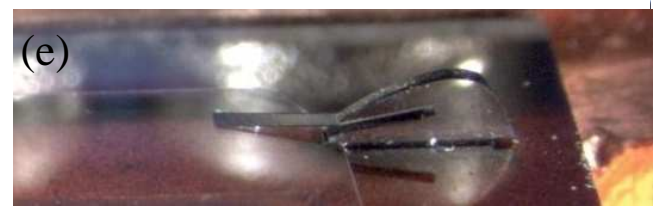

Figure 5: Summary on the design, implementation and characterization of the all-diamond assembly for the $100-\mu$ m-thick diamond plate. (a) Perspective view showing the components of the assembly: type IIa HPHT diamond (111) crystal plate 1, a CVD diamond substrate 2, diamond restrainers 3 , and perforated CVD diamond springs 4. (b) White-beam $\mathrm{X}$-ray topograph of the $100-\mu \mathrm{m}$-thick diamond crystal plate. The image is obtained from the $3 \overline{1} \overline{1}$ diamond reflection in Laue geometry. The dotted box illustrates the $5 \times 2 \mathrm{~mm}^{2}$ working regions containing only a few defects. (c) Image of the all-diamond assembly for the 100- $\mu \mathrm{m}$-thick diamond plate using CVD diamond springs with strain-relief cuts. The bright rectangle is the $5 \times 2 \mathrm{~mm}^{2}$ window in the CVD substrate for passage of X-rays transmitted through the diamond plates. (d) An enlarged view of the perforated CVD. The length of the spring is $\simeq 2 \mathrm{~mm}$ and the width is $0.3 \mathrm{~mm}$. (e) A side perspective view of a diamond restrainer. A CVD spring that acts on the HPHT crystal is inserted into a groove in the restrainer.

With these assemblies installed in the double-crystal monochromator at the LCLS, the capability of splitting the XFEL beam into a pink (transmitted) and a monochromatic (reflected) branch was successfully demonstrated as well as the use of the XFEL beam in two experiments simultaneously [8]. The averaged spectrum of the transmitted branch was evaluated using a bent crystal single-shot spectrometer [40]. The monochromatic branch was characterized at $7.9 \mathrm{keV}$ using a Si (440) crystal as an analyzer and by the rocking curve of the second (300- $\mu$ m-thick) diamond crystal. Nearly theoretical values were demonstrated. The throughput of the monochromator in the pink and the monochromatic branches was measured and was also found to be close to the expected theoretical values. In addition, temperature distribution in the all-diamond assembly of the first crystal was evaluated under the operating conditions using an infrared camera. It was found that the temperature variation in the all-diamond assembly does not exceed $\approx 3 \mathrm{C}^{\circ}$ 
while temperature distribution in the crystal remains fairly uniform.

The heat load is not a primary concern for diamond optics illuminated with the X-ray beam generated by the LCLS, where the average incident power density is $\approx 2 \mathrm{~W} / \mathrm{mm}^{2}$. Typical SASE X-ray pulse energy is $2-3 \mathrm{~mJ}$ at the repetition rate of $120 \mathrm{~Hz}$. In contrast, the power density incident on high-heat-load diamond optics at third-generation synchrotron sources is about $50-100 \mathrm{~W} / \mathrm{mm}^{2}$. At these power densities a substantial variation of temperature across the diamond plate in the all-diamond assembly (up to $\approx 50-100 \mathrm{~K})$ is expected as demonstrated by preliminary finite-element simulations [41].

The all-diamond assembly is not yet a universal solution to mounting of diamond crystals for application in all types of front-end synchrotron and XFEL optics. Preliminary experiments on the use of the assembly holding a 300- $\mu$ m-thick (111) diamond crystal revealed unstable performance possibly due to limited heat transfer between the diamond plate and the CVD substrate and/or by charge accumulation due to emission of photoelectrons. It is noted here that the presently widely accepted approach of mounting diamond crystals in the high-heat-load monochromators (e.g., [42]) involves diamond coupling to a cooled metallic block by means of InGa eutectic alloy which is a viscous liquid at room temperature. It is generally accepted that the main functions of the eutectic are to provide heat transfer and to immobilize the diamond (to provide angular stability) while keeping it strain-free. At the same time, an alternative solution is desirable since the eutectic can cause contamination and/or damage of the diamond if accidentally struck by the intense incident X-ray beam. Here it is noted that the eutectic also provides electrical grounding of the crystal which might help to compensate the photoemission-induced charge. As an alternative, grounded electrodes deposited on the diamond crystal can play this role. The use of eutectic is avoided in the mounting solution offered by the all-diamond assembly, however, a detailed study of heat transfer in the crystal-substrate interface is required for application in high-heat-load monochromators at 3rd and 4th generation synchrotrons and high-repetition-rate XFELs (X-ray sources now either planned or in construction). Further improvements in the design of the all-diamond assembly is work in progress at the Advanced Photon Source.

\subsection{High-energy-resolution optics with high efficiency}

The smallness of the intrinsic energy and angular widths of the Bragg reflections of diamond can be considered as an advantage for applications 
in high-energy-resolution X-ray optics combined with robustness of diamond due to its radiation hardness and high Young's modulus (resulting in high crystal stiffness).

Multicrystal X-ray monochromators with very small energy bandwidths are indispensable tools for inelastic X-ray scattering spectroscopy, nuclear resonant scattering spectroscopy, ultraprecise measurements of crystal lattice parameters, X-ray interferometry, X-ray metrology and for other applications in X-ray science. A conventional approach of monochromatization of hard $\mathrm{X}$-rays to relative energy bandwidths of $\Delta E / E \approx 10^{-5}$ and below relies on the use of high-order Bragg reflections. As a consequence X-ray monochromators with bandwidths $\Delta E<1 \mathrm{meV}$ have low spectral efficiencies of a few percent. An alternative approach to monochromatization of hard X-rays is the use of angular dispersive (AD) X-ray optics including schemes with strongly asymmetric Bragg crystals (dispersing elements) where the angle between the reflecting atomic planes and the crystal surface approaches $90^{\circ}$. The dispersing element transforms the spectral spread of X-rays into angular spread. A subsequent reflection acts as a wavelength selector, selecting the waves within a desired angular, and thus spectral interval [43]. Using diamond as a collimator/wavelength selector crystal the design of all-silicon AD optical schemes demonstrated earlier [44, 45] was optimized to yield an $\mathrm{X}$-ray monochromator with an unmatched performance in terms of spectral efficiency (65\%), and energy bandwidth (0.25 meV) [1].

A simplified optical scheme of the monochromator in the scattering plane is shown in Fig. 6(a). In the first Bragg reflection (diamond 133) the diamond crystal serves as a collimator of the incident X-ray beam. The reflected beam is dispersed using a strongly asymmetric $\mathrm{Si}_{1}$ (008) crystal and transmitted through the diamond crystal in normal transmittance (avoiding the anomalous transmission condition). The transmitted X-ray beam is then reflected from the second dispersing element $\mathrm{Si}_{2}$ (008) which results in an enhancement of angular dispersion. Finally, the same diamond crystal (also using 133 reflection) performs wavelength selection to $0.25 \mathrm{meV}$ bandwidth.

The diamond crystal used in this project was cut by a laser from the (001) growth sector and mechanically polished to fabricate a crystal plate with thickness of about $100 \mu \mathrm{m}$. A miscut angle $\alpha$ was deliberately introduced as shown in Fig.7(a) to achieve the required asymmetry for the working 133 reflection. Fig.7(b) shows a photograph of the diamond crystal and Fig.7(c) shows X-ray Lang topograph obtained using diamond 220 reflection in Laue geometry. The latter confirms high crystal quality (absence of defects in the 


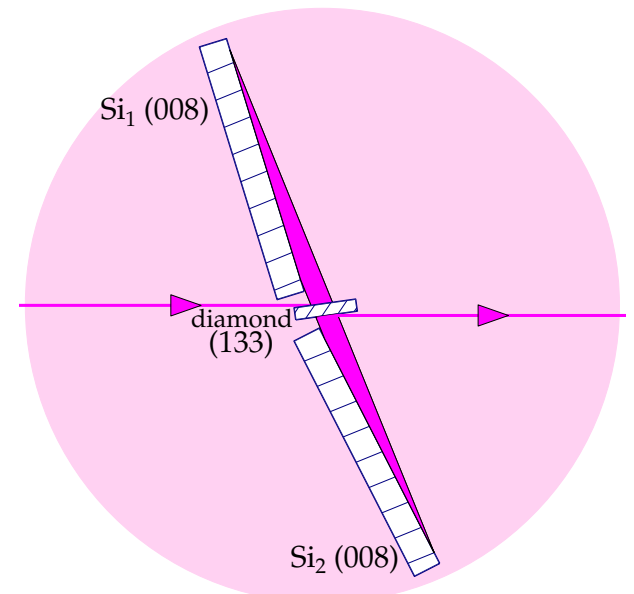

Figure 6: A simplified optical scheme of the monochromator (see text for details)

working region and beyond).

The monochromator utilizes X-rays with a photon energy of $\approx 9 \mathrm{keV}$. Therefore, it is applicable both at high-energy and at medium-energy storage ring synchrotron radiation facilities, as well as at XFEL facilities. Due to the choice of a thin diamond crystal as the primary crystal, operation of the monochromator is feasible with the self-seeded incident XFEL beam in the beam-multiplexing regime.

Typical power density of unfocused monochromatic beams (e.g, after a high-heat-load double-crystal monochromator at an undulator beamline of a third generation synchrotron) is $\approx 10 \mathrm{~mW} / \mathrm{mm}^{2}$. This beam is further monochromatized using high-energy-resolution optical schemes where diamond can serve as the first entrance crystal (as in the discussed hybrid diamond-silicon monochromator). Although, at such power density no radiation damage is expected for the diffracting diamond crystal, degradation of the mounting structure may become an issue. Figure 8(a) shows a picture of a simple mounting scheme for the diamond crystal in the hybrid monochromator before exposure to X-rays. The diamond crystal is supported on the edges by metallic stripes $(\approx 150-\mu \mathrm{m}$-thick steel plates). Figure $8(\mathrm{~b})$ shows the same arrangement after a few hours of exposure to a monochromatic X-ray beam with power density of about $15 \mathrm{~mW} / \mathrm{mm}^{2}$. Signs of corrosion are clearly seen in one of the supporting plates. During the exposure the performance of the monochromator was found unstable. This was caused by disturbance 

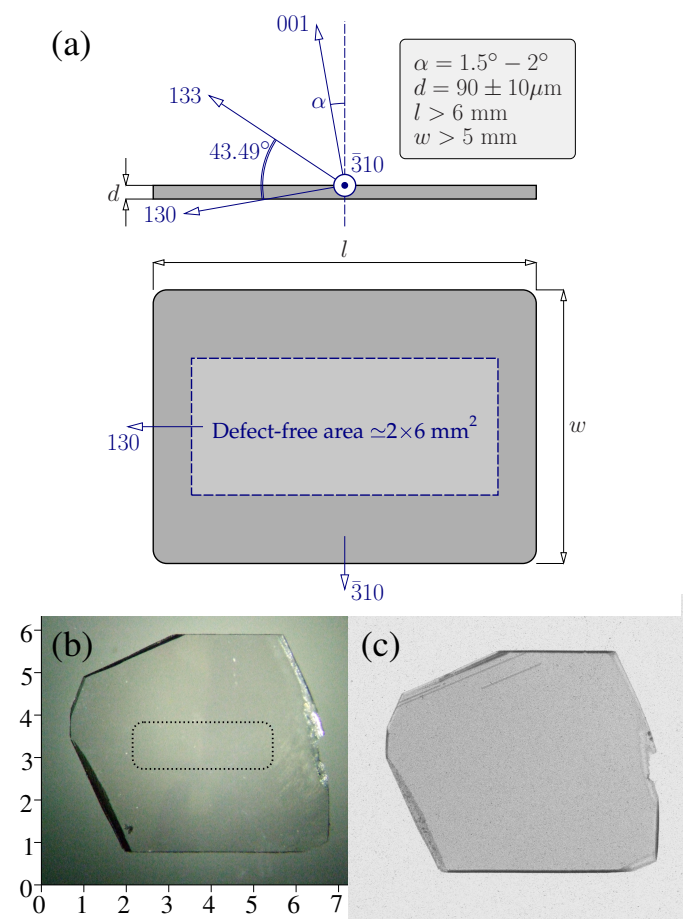

Figure 7: (a) Sketch of the crystal showing the requirements on diamond (133) crystal geometry and orientation. The arrows and Miller indexes denote reference reciprocal vectors. The miscut angle $\alpha=1.5^{\circ}$ from the (001) crystal plane yields the desired asymmetry angle $\eta_{\mathrm{C}}=48^{\circ}$ for the working diamond 133 Bragg reflection at a photon energy of $9.13 \mathrm{keV}$. (b) A photograph of the diamond crystal with dimensions scale given in $\mathrm{mm}$. The dashed box represents the maximum size of a footprint of an X-ray beam in the working configuration of the monochromator. (c) X-ray Lang topograph of the diamond crystal obtained using C 220 reflection in transmission (Laue) geometry. Crystal orientation in (b) and (c) is the same as in (a) (top view). 


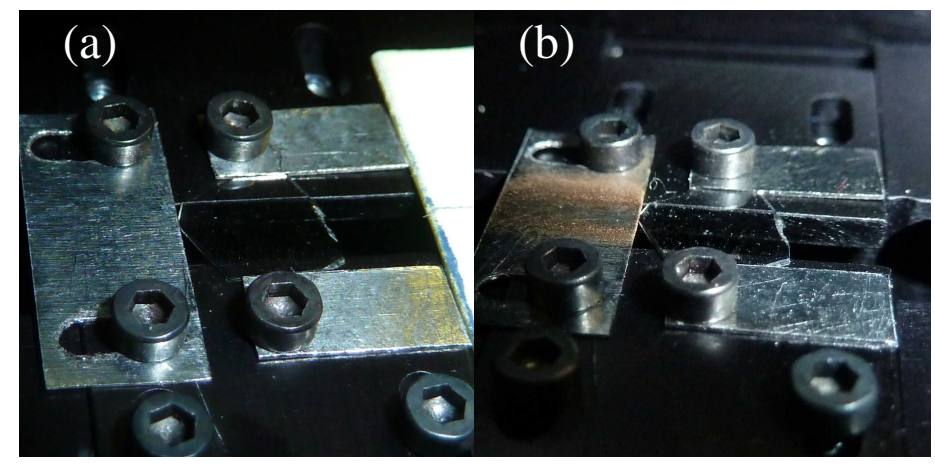

Figure 8: (a) A simple mounting scheme for the diamond crystal in the hybrid monochromator before exposure to X-rays. The diamond crystal is supported on the edges by metallic stripes $(\approx 150-\mu \mathrm{m}$-thick steel plates). (b) The same arrangement after a few hours of exposure to a monochromatic X-ray beam with power density of about $15 \mathrm{~mW} / \mathrm{mm}^{2}$. Signs of corrosion are clearly seen in one of the supporting plates.

of the angular position of the diamond crystal due to development of radiation damage in the supporting steel plate. Thus, the arrangement involving the steel plates was found unsuitable. The metallic plates were eventually replaced by diamond plates of low crystal quality.

This example demonstrates that utilization of materials with high radiation hardness (i.e., less susceptible to radiation damage) is preferable in the supporting structure. Mounting of diamond crystals using the all-diamond assembly discussed in the previous subsection is presently considered for applications in high-energy-resolution X-ray optics. Other advanced optical schemes involving diamond can benefit from the crystal mounting solution developed for the beam multiplexing monochromator at the LCLS. A recently proposed split-delay line of a Michelson interferometer type relies on the use of diamonds as diffracting crystals [16]. The three-crystal optical scheme features a small $(\approx 100 \mathrm{meV})$ dual-energy splitting due to backscattering configuration. This in combination with the use of diamond crystals should significantly increase the efficiency of the optics compared to the available schemes (e.g.,[46]). Finally, the all-diamond assembly can be considered as a potential crystal mounting solution for what could become the most spectacular application of diamond in X-ray optics - Bragg mirrors in the optical cavity of the X-ray free-electron laser oscillator (XFELO) $[9,10]$. These are discussed in the following section. 


\subsection{Bragg mirrors for X-ray free-electron laser oscillators}

The principle of operation of the XFELO borrows from that of a conventional laser where photons are trapped in an optical cavity and pass through a lasing medium multiple times. The XFELO as well as the conventional laser are fully coherent sources. The XFELO was shown to be feasible using ultralow emittance electron bunches from an energy-recovery linac and a low-loss optical cavity constructed from high-reflectivity crystals [9].

In order to achieve lasing the combined round-trip losses in the XFELO cavity should be less than about $10 \%$. This condition is satisfied within the narrow intrinsic energy bandwidth of Bragg reflections of certain highreflectivity crystals. The reflectivity of a crystal in Bragg diffraction results from an interplay between X-ray absorption and rigidity of crystal lattice (the latter is often described using the Debye temperature). Diamond has a unique combination of the two which favors high reflectivity. In theory, reflectivity close-to $100 \%$ can be achieved even when X-rays are reflected backwards in Bragg diffraction (> 99\% for some reflections). These theoretical predictions have been confirmed experimentally $[5,6]$.

Time-dependent expansion of the crystal lattice may become an issue due to the heat load of the intracavity XFELO pulses. The upper limit requirement on thermal variation of the lattice parameter $a$ should be set as $1 / 10$ the relative energy width of the reflectivity curve $\varepsilon$ :

$$
\delta a / a=0.1 \varepsilon .
$$

On the other hand, if partially absorbed X-ray pulse increases crystal temperature by $\Delta T$

$$
\delta a / a \approx \alpha(T) \Delta T,
$$

where $\alpha(T)$ is the temperature-dependent thermal expansion coefficient of diamond. The intracavity XFELO pulse will contain about $N_{p}=1 \times 10^{11}$ photons per area $A \approx 100 \times 100 \mu \mathrm{m}^{2}$ at a photon energy of $\mathrm{E} \approx 15 \mathrm{keV}$. The energy density per pulse is then $D=E N_{p} / A \approx 2 \mathrm{~J} / \mathrm{cm}^{2}$. Assuming that all energy deposited by the pulse is converted to thermal energy (i.e., upper estimate)

$$
\Delta T \approx \frac{D \mu(E)}{c \rho}
$$

where $\rho$ is the density, $c$ is the specific heat capacity, and $\mu(E)$ is the energydependent X-ray absorption coefficient for diamond (Eq. 3 is valid for crystals 
Table 1: Coefficients of the empirical formula Eq. 4 obtained using least-squares simultaneous fit of all available data.

\begin{tabular}{cc}
\hline \hline$b=3.6(6) \times 10^{-14} K^{-4}$ & $T_{0}=212(24) K$ \\
$c=1.21(3) \times 10^{-11} K^{-3}$ & $\Delta T_{0}=47(5) K$ \\
\hline
\end{tabular}

with thicknesses $d \ll 1 / \mu(E))$. At room temperature $(c \simeq 0.5 \mathrm{~J} / \mathrm{gK}$ and $\alpha \simeq 1 \times 10^{-6} \mathrm{~K}^{-1}$ ) Eq. 3 yields $\Delta T \approx 2 \mathrm{~K}$ and Eq. 2 yields $\delta a / a \simeq 2 \times 10^{-6}$. For a very practical case of the 008 reflection, $\varepsilon \simeq 1 \times 10^{-6}$. Thus, the criterion given by Eq. 1 is not satisfied at room temperature.

A possible solution to this potential problem is maintaining the crystal at low temperatures where a substantial reduction in the thermal expansion coefficient is expected. Reliable data on the thermal expansion coefficient of diamond at temperatures below $100 \mathrm{~K}$ were obtained only recently [47, 48]. It has been experimentally verified that the thermal expansion coefficient can be as small as $2 \times 10^{-9} \mathrm{~K}^{-1}$ at $T \lesssim 40 \mathrm{~K}$.

Figure 9 (a) shows the measured linear thermal expansion coefficients of four different diamond crystals in the temperature range $10-300 \mathrm{~K}$. No substantial difference in the data corresponding to crystals from different manufacturers and/or different crystal types (type IIa vs. type Ia) was found. All data combined were approximated by an empirical formula:

$$
\begin{gathered}
x(T)=b T^{3} W(T)+c T^{2}(1-W(T)), \\
W(T)=\left(1+\exp \frac{T-T_{0}}{\Delta T_{0}}\right)^{-1},
\end{gathered}
$$

The parameters in Eq. 4 determined using least-squares fitting of all available data are given in Table 1. Deviations of the thermal expansion from the empirical formula $\Delta \alpha(T)$ are shown in Fig. 9(b) for each of the four samples. These deviations do not exceed $3 \times 10^{-8} \mathrm{~K}^{-1}$ over the studied temperature range.

It is noted here that Eq. 4 can be used to approximate the thermal expansion coefficient at higher temperatures $(T>300 \mathrm{~K})$. However, it overestimates the available experimental data (e.g., [49]). The discrepancy becomes about $10 \%$ at $T \approx 500 \mathrm{~K}$ and increases at higher temperatures.

Overall, the availability of experimental data on Bragg reflectivity and low-temperature thermal expansion coefficient of diamond facilitates the development of the XFELO optics. The data further confirm feasibility of the 

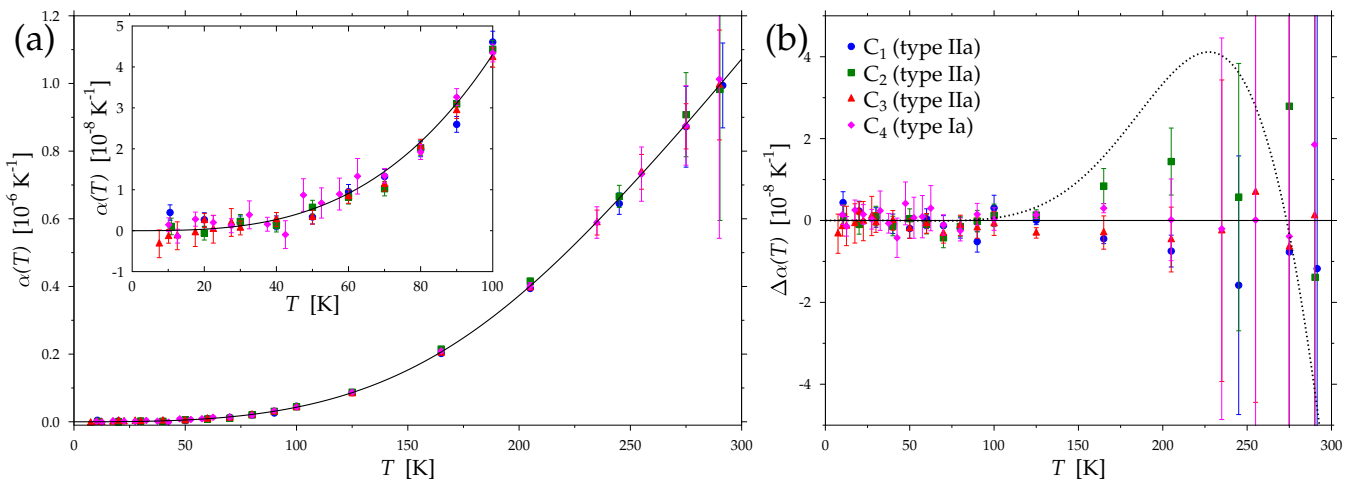

Figure 9: (a) Linear thermal expansion coefficients of IIa type diamond crystals $\mathrm{C}_{1-3}$ and Ia type $\mathrm{C}_{4}$ obtained using point-by-point calculation from the results of the measurements. The notation of the symbols is the same as shown in the legend of the other figure panel. The solid line represents approximation with the empirical formula. The inset shows region of temperatures below $100 \mathrm{~K}$ in more detail. (b) Deviations of the measured thermal expansion coefficients from the empirical approximation. The dotted line is the difference between the empirical formula and the best fit to the Debye approximation $(\alpha(T)=$ $\left.4.25(2) \times 10^{-14} T^{3}\right)$.

XFELO and enable precise simulations of performance of the XFELO optical cavity.

Research on Bragg mirrors for the XFELO is ongoing at the Advanced Photon Source and other synchrotron and XFEL facilities. Potential problems that need to be addressed by theoretical and experimental studies include X-ray induced radiation damage (cummulative damage due repetitive exposure to intracativy XFELO pulses at $\approx 1 \mathrm{MHz}$ frequency), charge accumulation due to photoemission, mounting-induced crystal strain, and mechanical stability of the XFELO cavity.

\section{Conclusions and Outlook}

The production of single crystals of diamond for X-ray optics still remains challenging since only a few manufactures have the necessary high-pressure high-temperature capabilities to produce crystals of high quality. Nevertheless, a synergy of state-of-the-art HPHT crystal growth, diamond processing technologies, and X-ray characterization methods leads to advanced practical applications of diamond in X-ray optics, such as, the high-resolution monochromator with an unprecedented combination of sub-millielectronvolt bandwidth and high efficiency, and the beam multiplexing monochromator, 
which improves the capabilities of XFELs. The application of diamond as a self-seeding monochromator at the LCLS demonstrates that diamond Xray optics can enable novel X-ray sources with unprecedented characteristics. The next expected step in this direction is the realization of the X-ray free-electron laser oscillator as the ultimate source for high-resolution X-ray spectroscopies.

The novel X-ray diamond crystal optics summarized in this work relies on availability of dislocation-free crystal regions with absence of growth striations and micro inclusions which ensures a good agreement with the theory in terms of Bragg reflectivity and the intrinsic energy/angular widths of the Bragg reflections. The ongoing progress on realization of X-ray sources with smaller angular divergence (better transverse coherence) implies reduction of $\mathrm{X}$ ray beam cross sections $\left(1 \times 1 \mathrm{~mm}^{2}\right.$ and less at practical distances from the source). For diffraction geometries close to backscattering (as well as for normal incidence transmission type optics) perfect diamond crystal regions can be as small as the beam cross section. For moderate Bragg angles it is expected that perfect regions of $1 \times 4 \mathrm{~mm}^{2}$ (with the largest dimension in the scattering plane) are sufficient to accommodate the entire beam footprint.

The requirement on the thickness of the perfect crystal region depends on the particular application. In the Bragg cases of X-ray diffraction ${ }^{1}$ utilized in the described novel applications, the characteristic penetration depth of $\mathrm{X}$-rays into the crystal (i.e., extinction depth) varies from about $1 \mu \mathrm{m}$ for low order reflections to about $100 \mu \mathrm{m}$ for practical high-order reflections. The reflectivity curve of a crystal with a thickness of about 10 extinction depths and greater is practically indistinguishable from that of an infinitely thick crystal. From this theoretical perspective, for the diamond 111 reflection (extinction depth $\approx 1 \mu \mathrm{m}$ ) the crystal thickness of about $10 \mu \mathrm{m}$ should be sufficient to form the optimal (thick-crystal limit) reflectivity curve. However, other factors such as mountinginduced and heat-load-induced crystal strains gain more importance since the stiffness of such an ultrathin crystal is dramatically

\footnotetext{
${ }^{1}$ In the Bragg case the entrance surface for the incident wave and the exit surface for the reflected wave is the same crystal surface.
} 
reduced. In addition, crystal warping may occur (e.g.,[50]). It is hoped that these problems can be resolved using a careful design and fabrication approach. Strain-free ultrathin perfect diamond crystals (thicknesses $\lesssim 50 \mu \mathrm{m}$ ) are highly demanded to further improve transmittance of the beam-multiplexing XFEL optics.

Finally, surface quality is as important for diamond crystal optics as for any other X-ray crystal optics. Far from grazing incidence (where the Bragg angle substantially exceeds the critical glancing angle for total external reflection) a simple criterion can be devised based on the refraction of X-rays on a wedge-shaped surface feature. A single ray passing through the wedge changes its direction of propagation by an angular amount $\Delta \psi \simeq \delta \Delta \alpha$, where $\Delta \alpha$ is the wedge angle and $\delta$ is the refraction correction ${ }^{2}$ (see e.g., [51]). The criterion can be based on the requirement that the angular deflection due to refraction on surface inhomogeneities (approximated by the wedge-shaped features) should not exceed 1/10 of the angular divergence of the incident $\mathbf{X}$-ray beam $\Delta \theta$. For example, the surface slope error $\Delta \alpha$ over the size of interest should not exceed $10 \mathrm{mrad}$ r.m.s. for an XFEL source $(\Delta \theta \approx 1 \mu$ rad r.m.s. $)$ at photon energies of about $8 \mathrm{keV}\left(\delta \simeq 1 \times 10^{-5}\right)$. If the size of interest is set to $\Delta x \simeq 1 \mu \mathrm{m}$, which is typical for X-ray spectroscopy and microscale imaging, the requirement on the surface roughness is $\Delta s=\Delta \alpha \Delta x \simeq 10 \mathrm{~nm}$ r.m.s.. Accordingly, imaging at the nanoscale (e.g., $\Delta x \approx 10 \mathrm{~nm}$ ) would require surface tolerances of a few $\AA$ [17].

In summary, the main targets for further development of diamond technology for X-ray crystal optics include defect-free crystal regions of sizes comparable with the sizes of X-ray beams (a few $\mathrm{mm}^{2}$ ) generated at synchrotron undulator beamlines and XFELs, crystals with thicknesses of $\lesssim 50 \mu \mathrm{m}$ without intrinsic strain and protected from the induced strains (due to mounting, heat load, vibrations, etc.), and, ultimately, a surface roughness of a few $\AA$.

\footnotetext{
${ }^{2}$ The refractive index for X-rays can be expressed as $n=1-\delta-i \beta$, where $\delta$ is a very small energy-dependent quantity $\left(\delta \approx 1 \times 10^{-5}-1 \times 10^{-6}\right.$ for hard X-rays, and $\left.\beta \ll \delta\right)$.
} 


\section{Acknowledgments}

Yu.V. Shvyd'ko, D. Shu, S.A. Terentyev and V.D. Blank are acknowledged for years of fruitful collaboration on diamond X-ray optics projects. Members of the XPP instrument team at the LCLS are acknowledged for input on performance of diamond optics at the LCLS. Members of the Optics group at the Advanced Photon Source are acknowledged for helpful discussions and for technical support. Use of the Advanced Photon Source was supported by the U.S. Department of Energy, Office of Science, under Contract No. DE-AC02-06CH11357.

\section{References}

[1] S. Stoupin, Yu. V. Shvyd'ko, D. Shu, V. D. Blank, S. A. Terentyev, S. N. Polyakov, M. S. Kuznetsov, I. Lemesh, K. Mundboth, S. P. Collins, J. P. Sutter, M. Tolkiehn, Hybrid diamond-silicon angular-dispersive Xray monochromator with 0.25 -mev energy bandwidth and high spectral efficiency, Opt. Express 21 (25) (2013) 30932-30946.

[2] S. Stoupin, S. A. Terentyev, V. D. Blank, Yu. V. Shvyd'ko, K. Goetze, L. Assoufid, S. N. Polyakov, M. S. Kuznetsov, N. V. Kornilov, J. Katsoudas, R. Alonso-Mori, M. Chollet, Y. Feng, J. M. Glownia, H. Lemke, A. Robert, S. Song, M. Sikorski, D. Zhu, All-diamond optical assemblies for a beam-multiplexing X-ray monochromator at the Linac Coherent Light Source, J. Appl. Cryst. 47 (2014) 1329-1336.

[3] J. Amann, W. Berg, V. Blank, F.-J. Decker, Y. Ding, P. Emma, Y. Feng, J. Frisch, D. Fritz, J. Hastings, Z. Huang, J. Krzywinski, R. Lindberg, H. Loos, A. Lutman, H.-D. Nuhn, D. Ratner, J. Rzepiela, D. Shu, Yu. Shvyd'ko, S. Spampinati, S. Stoupin, S. Terentiev, E. Trakhtenberg, D. Walz, J. Welch, J. Wu, A. Zholents, D. Zhu, Demonstration of self-seeding in a hard-X-ray free-electron laser, Nat. Photon. 6 (10) (2012) 693-698.

[4] S. Stoupin, V. Blank, S. Terentyev, S. Polyakov, V. N. Denisov, M. Kuznetsov, Yu. V. Shvyd'ko, D. Shu, P. Emma, J. Maj, J. Katsoudas, Diamond crystal optics for self-seeding of hard X-rays in X-ray free-electron lasers, Diamond and Related Materials 33 (0) (2013) 1 - 4. 
[5] Yu. V. Shvyd'ko, S. Stoupin, A. Cunsolo, A. Said, X. Huang, Highreflectivity high-resolution X-ray crystal optics with diamonds, Nat. Phys. 6 (3) (2010) 196.

[6] Yu. V. Shvyd'ko, S. Stoupin, V. Blank, S. Terentyev, Near 100\% Bragg reflectivity of X-rays, Nat. Photon. 5 (2011) 539.

[7] Y. Feng, R. Alonso-Mori, V. Blank, S. Boutet, M. Chollet, T. B. van Driel, D. M. Fritz, J. M. Glownia, J. B. Hastings, H. Lemke, M. Messerchmidt, P. A. Montanez, A. Robert, J. Robinson, L. Samoylova, Yu. Shvydko, M. Sikorski, H. Sinn, S. Song, V. N. Srinivasan, S. Stoupin, S. Terentiev, G. Williams, D. Zhu, Recent development of thin diamond crystals for X-ray FEL beam-sharing, Proc. SPIE, Advances in X-ray Free-Electron Lasers II: Instrumentation 8778 (2013) 87780B.

[8] D. Zhu, Y. Feng, S. Stoupin, S. A. Terentyev, H. T. Lemke, D. M. Fritz, M. Chollet, J. M. Glownia, R. Alonso-Mori, M. Sikorski, S. Song, T. B. van Driel, G. J. Williams, M. Messerschmidt, S. Boutet, V. D. Blank, Yu. V. Shvyd'ko, A. Robert, Performance of a beam-multiplexing diamond crystal monochromator at the Linac Coherent Light Source, Rev. Sci. Instrum. 85 (6) (2014) 063106.

[9] K.-J. Kim, Yuri. Shvyd'ko, S. Reiche, A proposal for an X-ray freeelectron laser oscillator with an energy-recovery linac, Phys. Rev. Lett. 100 (24) (2008) 244802.

[10] K.-J. Kim, Y. V. Shvyd'ko, Tunable optical cavity for an X-ray freeelectron-laser oscillator, Phys. Rev. ST Accel. Beams 12 (3) (2009) 030703.

[11] D. Shu, Y. Shvyd'ko, J. Amann, P. Emma, S. Stoupin, J. Quintana, Design of a diamond-crystal monochromator for the LCLS hard X-ray self-seeding project, Journal of Physics: Conference Series 425 (5) (2013) 052004 .

[12] G. Grübel, D. Abernathy, G. Vignaud, M. S. del Rio, A. Freund, A diamond double-crystal transmission monochromator for the TROIKA II station at ESRF, Rev. Sci. Instrum. 67 (9) (1996) 3349-3349.

[13] P. Fernandez, T. Graber, W.-K. Lee, D. Mills, C. Rogers, L. Assoufid, Test of a high-heat-load double-crystal diamond monochromator at the 
advanced photon source, Nucl. Instrum. Methods Phys. Res. A 400 (23) (1997) $476-483$.

[14] M. Yabashi, S. Goto, Y. Shimizu, K. Tamasaku, H. Yamazaki, Y. Yoda, M. Suzuki, Y. Ohishi, M. Yamamoto, T. Ishikawa, Diamond doublecrystal monochromator for SPring8 undulator beamlines, AIP Conf. Proc. 879 (1) (2007) 922-925.

[15] Yu. Shvydko, S. Stoupin, D. Shu, S. P. Collins, K. Mundboth, J. Sutter, M. Tolkiehn, High-contrast sub-millivolt inelastic Xray scattering for nano- and mesoscale science, Nat. Commun. 5. doi:10.1038/ncomms5219.

[16] Yu. P. Stetsko, Yu. V. Shvyd'ko, G. Brian Stephenson, Time-delayed beam splitting with energy separation of X-ray channels, Appl. Phys. Lett. 103 (17) (2013) 173508.

[17] R. C. Burns, A. I. Chumakov, S. H. Connell, D. Dube, H. P. Godfried, J. O. Hansen, J. Härtwig, J. Hoszowska, F. Masiello, L. Mkhonza, M. Rebak, A. Rommevaux, R. Setshedi, P. V. Vaerenbergh, Hpht growth and X-ray characterization of high-quality type IIa diamond, J. Phys.: Condensed Matter 21 (36) (2009) 364224.

[18] C. Giles, C. Malgrange, J. Goulon, F. de Bergevin, C. Vettier, E. Dartyge, A. Fontaine, C. Giorgetti, S. Pizzini, Energy-dispersive phase plate for magnetic circular dichroism experiments in the X-ray range, J. Appl. Cryst. 27 (3) (1994) 232-240.

[19] K. Hirano, T. Ishikawa, S. Kikuta, Development and application of xray phase retarders (invited), Rev. Sci. Instrum. 66 (2) (1995) 1604-1609.

[20] L. E. Berman, Q. Shen, K. D. Finkelstein, P. Doing, Z. Yin, G. Pan, Characterization of a diamond crystal X-ray phase retarder, Rev. Sci. Instrum. 73 (3) (2002) 1502-1504.

[21] M. Suzuki, Y. Inubushi, M. Yabashi, T. Ishikawa, Polarization control of an X-ray free-electron laser with a diamond phase retarder, J. Synchrotron Rad. 21 (3) (2014) 466-472. 
[22] F. Bundy, W. Bassett, M. Weathers, R. Hemley, H. Mao, A. Goncharov, The pressure-temperature phase and transformation diagram for carbon; updated through 1994, Carbon 34 (2) (1996) 141 - 153.

[23] H. Sumiya, K. Tamasaku, Large defect-free synthetic type IIa diamond crystals synthesized via high pressure and high temperature, Japn. J. Appl. Phys. 51 (2012) 090102.

[24] A. M. Zaitsev, Optical properties of diamond: a data handbook, Springer, Berlin, 2001.

[25] P. M. Martineau, M. P. Gaukroger, K. B. Guy, S. C. Lawson, D. J. Twitchen, I. Friel, J. O. Hansen, G. C. Summerton, T. P. G. Addison, R. Burns, High crystalline quality single crystal chemical vapour deposition diamond, J. Phys.: Condensed Matter 21 (36) (2009) 364205.

[26] M. Moore, Imaging diamond with X-rays, J. Phys.: Condensed Matter 21 (36) (2009) 364217.

[27] A. R. Lang, A. P. W. Makepeace, M. Moore, W. C. Machado, On the variation of X-ray diffraction contrast with wavelength: A study with synchrotron radiation, J. Appl. Cryst. 16 (1) (1983) 113-125.

[28] K. Tamasaku, T. Ueda, D. Miwa, T. Ishikawa, Goniometric and topographic characterization of synthetic IIa diamonds 38 (10A) (2005) A61.

[29] S. Polyakov, V. Denisov, N. Kuzmin, M. Kuznetsov, S. Martyushov, S. Nosukhin, S. Terentiev, V. Blank, Characterization of top-quality type IIa synthetic diamonds for new X-ray optics, Diamond and Related Materials 20 (5-6) (2011) $726-728$.

[30] B. Nöhammer, J. Hoszowska, A. K. Freund, C. David, Diamond planar refractive lenses for third- and fourth-generation X-ray sources, J. Synchrotron Rad. 10 (2) (2003) 168-171.

[31] A. F. Isakovic, A. Stein, J. B. Warren, S. Narayanan, M. Sprung, A. R. Sandy, K. Evans-Lutterodt, Diamond kinoform hard X-ray refractive lenses: design, nanofabrication and testing, J. Synchrotron Rad. 16 (1) (2009) 8-13. 
[32] L. Alianelli, K. J. S. Sawhney, A. Malik, O. J. L. Fox, P. W. May, R. Stevens, I. M. Loader, M. C. Wilson, A planar refractive X-ray lens made of nanocrystalline diamond, J. Appl. Phys. 108 (12) (2010) 123107.

[33] C. David, S. Gorelick, S. Rutishauser, J. Krzywinski, J. Vila-Comamala, V. A. Guzenko, O. Bunk, E. Farm, M. Ritala, M. Cammarata, D. M. Fritz, R. Barrett, L. Samoylova, J. Grunert, H. Sinn, Nanofocusing of hard X-ray free electron laser pulses using diamond based fresnel zone plates, Sci. Rep. 1 (2011) 57.

[34] F. Uhlén, S. Lindqvist, D. Nilsson, J. Reinspach, U. Vogt, H. M. Hertz, A. Holmberg, R. Barrett, New diamond nanofabrication process for hard X-ray zone plates, J. Vac. Sci. Technol. B 29 (6) (2011) 06FG03.

[35] U. Flechsig, R. Abela, R. Betemps, H. Blumer, K. Frank, A. Jaggi, A. A. MacDowell, H. A. Padmore, V. Schnherr, J. Ulrich, H. Walther, S. Zelenika, C. Zumbach, The SLS optics beamline, AIP Conf. Proc. 879 (1) (2007) 890-893.

[36] J. Als-Nielsen, A. Freund, G. Grübel, J. Linderholm, M. Nielsen, M. del Rio, J. Sellschop, Multiple station beamline at an undulator X-ray source, Nucl. Instrum. Methods Phys. Res. B 94 (3) (1994) 306 - 318.

[37] G. Grübel, J. Als-Nielsen, A. Freund, The TROIKA beamline at ESRF, J. Phys. IV France 04 (C9) (1994) 27.

[38] P. Emma, R. Akre, J. Arthur, R. Bionta, C. Bostedt, J. Bozek, A. Brachmann, P. Bucksbaum, R. Coffee, F.-J. Decker, Y. Ding, D. Dowell, S. Edstrom, A. Fisher, J. Frisch, S. Gilevich, J. Hastings, G. Hays, P. Hering, Z. Huang, R. Iverson, H. Loos, M. Messerschmidt, A. Miahnahri, S. Moeller, H.-D. Nuhn, G. Pile, D. Ratner, J. Rzepiela, D. Schultz, T. Smith, P. Stefan, H. Tompkins, J. Turner, J. Welch, W. White, J. Wu, G. Yocky, J. Galayda, First lasing and operation of an angstromwavelength free-electron laser, Nat. Photon. 4 (9) (2010) 641-647.

[39] T. Ishikawa, H. Aoyagi, T. Asaka, Y. Asano, N. Azumi, T. Bizen, H. Ego, K. Fukami, T. Fukui, Y. Furukawa, S. Goto, H. Hanaki, T. Hara, T. Hasegawa, T. Hatsui, A. Higashiya, T. Hirono, N. Hosoda, M. Ishii, T. Inagaki, Y. Inubushi, T. Itoga, Y. Joti, M. Kago, T. Kameshima, H. Kimura, Y. Kirihara, A. Kiyomichi, T. Kobayashi, C. Kondo, 
T. Kudo, H. Maesaka, X. M. Marechal, T. Masuda, S. Matsubara, T. Matsumoto, T. Matsushita, S. Matsui, M. Nagasono, N. Nariyama, H. Ohashi, T. Ohata, T. Ohshima, S. Ono, Y. Otake, C. Saji, T. Sakurai, T. Sato, K. Sawada, T. Seike, K. Shirasawa, T. Sugimoto, S. Suzuki, S. Takahashi, H. Takebe, K. Takeshita, K. Tamasaku, H. Tanaka, R. Tanaka, T. Tanaka, T. Togashi, K. Togawa, A. Tokuhisa, H. Tomizawa, K. Tono, S. Wu, M. Yabashi, M. Yamaga, A. Yamashita, K. Yanagida, C. Zhang, T. Shintake, H. Kitamura, N. Kumagai, A compact X-ray free-electron laser emitting in the sub-angstrom region, Nat. Photon. 6 (8) (2012) 540-544.

[40] D. Zhu, M. Cammarata, J. M. Feldkamp, D. M. Fritz, J. B. Hastings, S. Lee, H. T. Lemke, A. Robert, J. L. Turner, Y. Feng, A singleshot transmissive spectrometer for hard X-ray free electron lasers, Appl. Phys. Lett. 101 (3) (2012) 034103.

[41] Terentyev, et al., (unpublished) (2013).

[42] W. Schildkamp, Monochromator crystal mounting stage, Rev. Sci. Instrum. 73 (3) (2002) 1571-1573.

[43] Yu. Shvyd'ko, X-Ray Optics - High-Energy-Resolution Applications, Vol. 98 of Optical Sciences, Springer, Berlin Heidelberg New York, 2004.

[44] Yu. Shvyd'ko, S. Stoupin, D. Shu, R. Khachatryan, Using angular dispersion and anomalous transmission to shape ultramonochromatic $\mathrm{x}$ rays, Phys. Rev. A 84 (2011) 053823.

[45] Yu. Shvyd'ko, S. Stoupin, K. Mundboth, J. Kim, Hard-X-ray spectrographs with resolution beyond $100 \mu \mathrm{eV}$, Phys. Rev. A 87 (2013) 043835.

[46] W. Roseker, H. Franz, H. Schulte-Schrepping, A. Ehnes, O. Leupold, F. Zontone, A. Robert, G. Grübel, Performance of a picosecond X-ray delay line unit at $8.39 \mathrm{kev}$, Opt. Lett. 34 (12) (2009) 1768-1770.

[47] S. Stoupin, Yu.. V. Shvyd'ko, Thermal expansion of diamond at low temperatures, Phys. Rev. Lett. 104 (2010) 085901.

[48] S. Stoupin, Yu.. V. Shvyd'ko, Ultraprecise studies of the thermal expansion coefficient of diamond using X-ray diffraction in backscattering, Phys. Rev. B 83 (2011) 104102. 
[49] Handbook of industrial diamonds and diamond films, Marcel Dekker, Inc., New York, 1998.

[50] G. Yang, R. Jones, F. Klein, K. Finkelstein, K. Livingston, Rocking curve imaging for diamond radiator crystal selection, Diamond and Related Materials 19 (79) (2010) 719 - 722.

[51] J. Als-Nielsen, D. McMorrow, Elements of Modern X-ray Physics, John Wiley \& Sons, Ltd, New York Chichester Weinheim Brisbane Singapore Toronto, 2011. 